\title{
Influence of Fluctuating Pulse Durations on the Time Resolution of Probing Experiments
}

\author{
W. Zinth \\ Physik Department, Technische Universität, D-8000 München, Fed. Rep. Germany
}

Received 9 August 1981/Accepted 28 August 1981

\begin{abstract}
Fluctuating pulse durations considerably influence the time resolution of probing experiments. The commonly used arithmetic averaging the experimental data changes the shape of the signal curves and gives longer decay times in the wings. Methods are presented to reduce the effect of fluctuating pulse durations.
\end{abstract}

PACS: $06,42.80$

With the advent of picosecond light pulses the investigation of ultrashort dynamical processes has become an active field of research. Various laser systems, mode-locked dye lasers, and Nd-glass lasers with pulse durations of the order of one picosecond have been employed to measure a variety of ultrashort phenomena $[1-3]$. For the experimentalist it is essential to know the time resolution of the system to state the shortest time constant he is able to measure. It has been recognized generally that the time resolution is determined by the duration and the shape of the light pulses. Little attention has been given to the influence of the fluctuations of the pulse duration on the time resolution of a specific experiment. Since all existing mode-locked laser systems generate ultrashort light pulses with a spread of pulse durations, a discussion of the effect on probing experiments appears to be appropriate.

Probing experiments are performed in the following way: An ultrashort light pulse is split into two parts. The first pulse is used to excite a material, whereas the second pulse monitors the time dependence of the material excitation. An optical delay line introduces a variable time separation $t_{D}$ between the excitation and probing pulse. The time dependence of the material excitation $M(t)$ is obtained when the signal $S\left(t_{D}\right)$ produced in the probing process is measured as a function of the time delay $t_{p}$. We briefly mention two examples for probing experiments: (i) "Exciting" and "probing" pulse cross within a nonlinear crystal under phase-matching conditions, and a second harmonic light is generated as long as the two pulses overlap. In this case, the material excitation $M(t)$ is proportional to the product of the exciting pulse intensity $I_{e}$ times the second order susceptibility $\chi^{(2)}$. The signal plotted versus the delay time reproduces the correlation function of the light pulses. It has been shown in a recent paper that the autocorrelation curve is strongly effected by the fluctuations of the pulse duration [4]. Care has to be taken to deduce a value for the pulse duration from the width of the correlation curve. (ii) A first pulse excites a molecular vibrational mode (e.g., by resonant infrared absorption) generating a population $M(t)$ in the upper vibrational level. A second pulse probes $M(t)$ by anti-Stokes Raman scattering. When the decay time $T$ of the population is longer than the pulse duration $t_{p}$ one readily measures $T$. When the time constant $T$ is much shorter than the pulse duration, the shape of the signal curve equals the correlation function between the exciting and the probing pulse. Molecular decay times can only be measured when the signal curve differs from the correlation curve. The shortest molecular time constant which can be measured depends on the slope (the "decay") of the correlation curve. For instance, using Gaussian shaped pulses one finds a better time resolution when data are taken far below the peak (down the wings). A factor of ten below the maximum one finds a slope of the autocorrelation curve of $\tau=0.28 t_{p}$, whereas at a factor of $10^{3}$ below the peak the slope is steeper, $\tau \simeq 0.17 t_{p}, t_{p}$ being the duration (FWHM) of the pulse. The question now arises on the time resolution when 


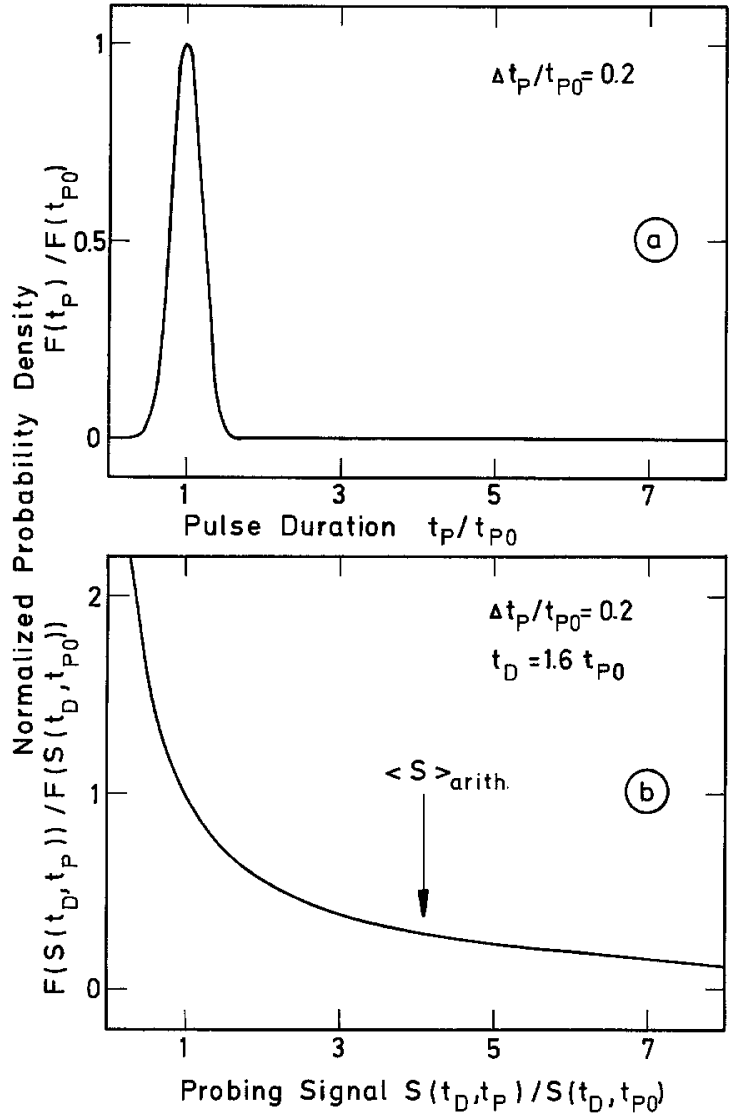

Fig. 1a and $b$. Normalized probability density functions for the pulse duration $t_{p}$ (a) and the probing signal (b). In both cases the same fluctuations of the pulse duration $\Delta t_{p} / t_{p 0}=0.2$ are assumed

pulses of fluctuating pulse durations are used in the experiments.

\section{Theoretical Model}

We begin the following discussion with a simplified system where the material excitation has the shape of a delta function, i.e. $M(t)=M_{0} \delta(t)$. This situation may be realized when the duration of the exciting pulse $t_{p e}$ is much shorter than the duration $t_{p}$ of the probing pulse and when the material excitation decays very rapidly, $T \ll t_{p}$. We further assume a linear interaction of the probing pulse intensity $I\left(t+t_{D}, t_{p}\right)$ with the material excitation $M(t)=M_{0} \delta(t)$. Experimentally we observe a time integrated signal:

$S\left(t_{D}, t_{p}\right) \propto \int_{-\infty}^{+\infty} d t M(t) I\left(t+t_{D}, t_{p}\right)=M_{0} I\left(t_{D}, t_{p}\right)$.

The signal measured as a function of the delay time reproduces the shape of the probing pulse and the time resolution is determined by its shape. When the pulse duration fluctuates, the signal $S\left(t_{D}, t_{p}\right)$ for a fixed delay time varies from shot to shot. It is general practice to simply average over many shots [5]. Near the peak of the signal curve fluctuating pulse durations do not change the signal considerably. But the rapidly decaying wings depend strongly on the pulse duration. Long pulses give very large signals and short pulses have signals close to zero. Under these conditions the averaging procedure is of paramount importance for the determination of the true shape of the signal curve. To illustrate the result of the commonly used averaging, the arithmetic mean, we calculate the probing signal $S\left(t_{D}, t_{p}\right)$ for fluctuating probing pulses of Gaussian shape. We assume a normal probability density $F\left(t_{p}\right)$ of the pulse duration $t_{p}$ fluctuating around the most probable pulse duration $t_{p 0}$.

$F\left(t_{p}\right)=\frac{1}{\Delta t_{p} \sqrt{2 \pi}} \exp \left|-\frac{1}{2}\left(\frac{t_{p}-t_{p 0}}{\Delta t_{p}}\right)^{2}\right|$.

The width of the frequency distribution is $\Delta t_{p}$, and more than $66 \%$ of all pulses have durations between $t_{p 0} \pm \Delta t_{p}$. The distribution is truncated at $t_{p}=0$.

In Fig. 1a we plot the probability density of (2) for $\Delta t_{p} / t_{p 0}=0.2$. Only pulse durations within a narrow range around $t_{p 0}$ may occur.

Next we calculate the probability density of the signal values $S$. The fluctuations of the pulse duration strongly influences the magnitude of the signals $S$ at a certain delay position $t_{D}$. For Gaussian shaped probing pulses we write for the signal $S$

$S\left(t_{D}, t_{p}\right) \propto \exp \left[-\left(t_{D} 2 \sqrt{\ln 2} / t_{p}\right)^{2}\right]$.

The probability density $F(S)$ of a certain signal value for fluctuating pulse durations is calculated from

$F\left(S\left(t_{D}, t_{p}\right)\right)=F\left(t_{p}\right) \times d t_{p} / d S$.

$F(S)$ was evaluated for the delay time $t_{D}=1.6 t_{p 0}$ (where the $S$-value is approximately $10^{-3}$ below the peak value at $t_{D}=0$ ) and for pulse fluctuations $\Delta t_{p} / t_{p 0}=0.2$. In Fig. $1 \mathrm{~b}$ the calculated curve $F(S)$ is presented. The probing signal is normalized to the signal value for the most frequent pulse duration $t_{p 0}, S\left(t_{D}, t_{p 0}\right)$. A high probability density $F\left(S\left(t_{D}, t_{p}\right)\right)$ is found for the small signal values. $F(S)$ decreases to higher signal values but high signals occur with non-vanishing probability. The influence of the high signals is readily seen when we calculate the arithmetic average (expectation value or arithmetic mean).

$\left\langle S\left(t_{D}\right)\right\rangle_{\text {arith }}=\int_{0}^{\infty} S F(S) d S=\int_{0}^{\infty} F\left(t_{p}\right) S\left(t_{D}, t_{p}\right) d t_{p}$.

For our example the average signal is found at $S\left(t_{D}\right)_{\text {arith }} \simeq 4.2 S\left(t_{D}, t_{p 0}\right)$; i.e., the averaged value is four times larger than the signal one would expect from the most probable pulse duration $t_{p 0}$. 


\section{Arithmetic Averaging}

The influence of the fluctuating pulse durations is obvious when we plot the arithmetic mean of the signal as a function of the delay time $t_{D}$ (Fig. 2). For pulses with a constant duration $t_{p 0}$ (i.e., for $\Delta t_{p}=0$ ) the signal curve has the shape of the Gaussian function (dashed curve in Fig. 2). For a situation with fluctuating pulse durations the calculated probe pulses, (5), give new signal curves. The solid curves of Fig. 2 are calculated for a width of the distribution of the pulses: $\Delta t_{p} / t_{p 0}=0.2,0.3$, and 0.5 . Two properties of the signal curves are striking: The fluctuations of $t_{p}$ lead to a more pronounced peak at $t_{D}=0$ and at later delay times $\left(t_{D}>0.5 t_{p 0}\right)$ they produce a slower decaying, nearly exponential, signal curve. At a signal level of $10^{-3}$ we find the following apparent time constants: $\tau=0.12 t_{p 0}$ for the constant pulse duration and longer time constants $\tau=0.17 t_{p 0}$ and $\tau=0.28 t_{p 0}$ when the pulse durations fluctuate with $\Delta t_{p} / t_{p 0}=0.2$ and 0.5 , respectively. For example, using a pulse distribution with $t_{p 0}=6 \mathrm{ps} \pm 3 \mathrm{ps}$ the apparent decay of the signal curve is increased by $240 \%$ from $0.7 \mathrm{ps}$ (without fluctuations) to $\tau=1.7 \mathrm{ps}$.

It should be emphasized that as a result of the averaging procedure Gaussian pulses give signal curves with exponential wings. A careful consideration of the pulse distribution is necessary in order not to confuse the exponential wings with a time constant of the material excitation. A distribution of pulse durations reduces the time resolution of the probing experiments substantially.

The arithmetic averaging discussed here is used in most experimental investigations. In the experiments one sums up the different signals (at a certain delay position) and normalizes to the total number of shots. In particular, mode-locked systems of high pulse repetition rate use detection systems with long time constants. In this way one obtains directly the arithmetic mean over many pulses of varying pulse duration.

\section{Central Point Averaging}

How can one reduce or overcome the problems resulting from fluctuating pulse durations? A straightforward technique consists of measuring the pulse duration of each pulse and taking the signal data for pulse durations within a narrow distribution. When only very short pulses are used the time resolution is optimized. This measuring technique is frequently complicated and time consuming.

We suggest here a different averaging procedure which yields data of high time resolution [6]. We first have to discuss the reason for the difficulties arising with the

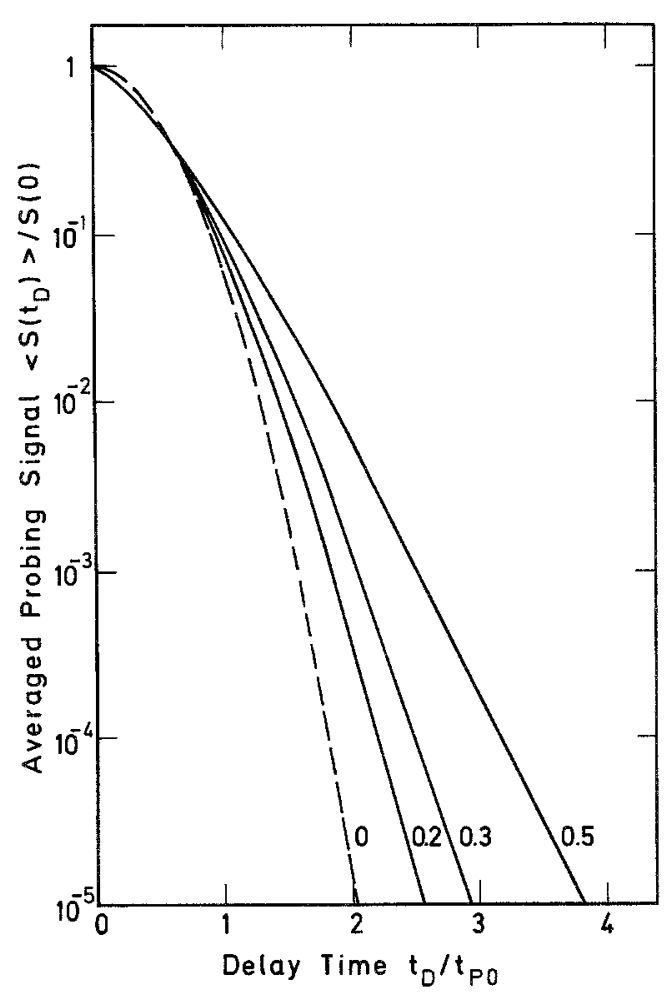

Fig. 2. Arithmetically averaged signals calculated for Gaussian shaped pulses probing a delta function shaped excitation. The peak intensity of the probing pulses is kept constant. (Broken curve: constant pulse duration $t_{p}=t_{p 0}$. Solid curves: fluctuations of the pulse duration $\Delta \mathrm{t}_{p} / \mathrm{t}_{p 0}=0.2,0.3$, and 0.5 )

arithmetic averaging. The shape of the averaged signal curve changes as the different pulse durations do not contribute equally at all the delay times to the arithmetic mean. At short delay times all the pulses contribute whereas in the wings especially the longer pulses with their large signals determine the arithmetic average. To find a better time resolution of the probing experiment one has to assure that only one narrow band of pulse durations is allowed to determine the mean signal value. When we do not want to measure the pulse durations we have to find out how to correlate specific signal values with specific pulse durations independent of the delay time.

We know that the signal is a monotoneous function of the pulse duration. Therefore, the probability to find signal values smaller than $S=S\left(t_{p}, t_{D}\right)$ (i.e., the distribution function) is equal to the probability to find pulse durations smaller than $t_{p}$. This relation allows us now to select specific pulses from a measured distribution of signal values; e.g., we may use the center $S_{c}$ of the signal distribution where we have as many smaller as larger signal values. We call this value the "central point" of the signal distribution. The above mentioned relation states that the signal $S_{c}$ is produced by the pulse with the duration $t_{p c}$, i.e. by the central point of 


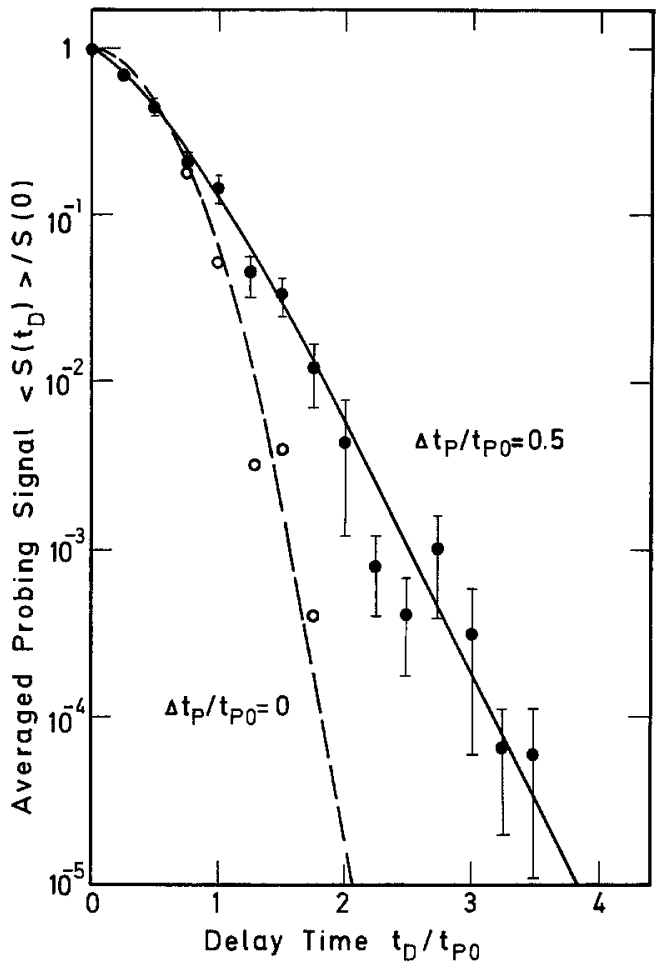

Fig. 3. Arithmetically averaged signals calculated for Gaussian pulses probing a delta function shaped excitation. (Broken curve: constant pulse duration; solid curve: $\Delta t_{p} / t_{p 0}=0.5$.) The full points indicate the arithmetic mean for 40 pulses with randomly distributed durations, (2). The circles show the "central points" of the signal distribution determined for the same set of pulse durations. This different procedure reproduces the correct shape of the signal curve

the distribution of the pulse durations. When we plot the central point of the signal as a function of the delay time this curve should coincide with the curve determined by the pulse duration $t_{p c}$. For the normal distribution used here, (2), the center value $t_{p c}$ equals the most probable pulse duration, $t_{p c}=t_{p 0}$. For a measurement with $N$ shots per delay time setting, $N \gg 1$, the central point of the signal is determined in the following way: The shots are numbered according to their increasing signal amplitudes. The signal of the $(N / 2)^{\text {th }}$ shot is called the central point. Using the central point of a distribution we overcome the difficulties of arithmetic averaging and may optimize the time resolution. This property is demonstrated in Fig. 3 where we compare the arithmetic mean and central point values for the example of fluctuating pulse durations.

The broken curve is calculated when all pulses have the same pulse duration $t_{p 0}$. The solid curve shows arithmetic averaging with $\Delta t_{p} / t_{p 0}=0.5,(5)$. For various delay times we generated in a random process 40 "pulses" distributed according to (2) with $\Delta t_{p} / t_{p 0}=0.5$ Using these pulses we calculated the arithmetic mean (full points in Fig. 3) and the standard deviation of the signal. The points are near the theoretical curve, showing the slower decay and the non-Gaussian shape typical for the arthmetic averaging. For the same set of pulse durations we determined the "central points" of the signal distribution (circles in Fig. 3). The "central point" lies close to the dashed curve calculated with $t_{p}=t_{p 0}$ and shows the correct Gaussian shape of the signal. The remaining small differences between the central points and the theoretical line for $t_{p}=t_{p 0}$ are due to statistical incertainties caused by the limited number of shots.

\section{Conclusion}

Decay times $T$ of the material excitation larger than the spread $\Delta t_{p}$ of the pulse duration, $T>\Delta t_{p}$ (for Gaussian shaped pulses) are not influenced by the arithmetic averaging process. For $T \leqq \Delta t_{p}$ arithmetical averaging of the probe signal leads to nearly exponentially decaying wings. Determining the central point of the signal distribution allows to overcome these difficulties. The signal curve is formed by pulses of a predicted, the central point, pulse duration. Using Gaussian shaped light pulses the time resolution is only limited by the amount of the useful signal amplitude, i.e., by the signal to noise ratio.

Acknowledgement. The author acknowledges valuable contributions and stimulating discussions with Professor W. Kaiser.

\section{References}

1. See, for example: Picosecond Phenomena II, ed. by R.M.Hochstrasser, W.Kaiser, and C.V.Shank, Springer Ser. Chem. Phys. 14 (Springer, Berlin, Heidelberg, New York 1980)

2. A.Laubereau, W.Kaiser: Rev. Mod. Phys. 50, 607 (1978)

3. C.V.Shank, R.L.Fork, F.R.Leheny, Jagdeep Shah: Phys. Rev. Lett. 42, 112 (1979)

4. E.W.van Stryland: Opt. Commun. 31, 93 (1979)

5. T.S.Durrani, C.A.Greated: Laser Systems in Flow Measurements (Plenum Press, New York 1977) p. 7

A.G.Worthing, J.Geffner: Treatment of Experimental Data (Wiley, New York 1955) p. 177

6. W.Zinth, H.-J.Polland, A.Laubereau, W.Kaiser: Appl. Phys. B26, $77-88$ (1981) 